\title{
EL TRABAJO COOPERATIVO ARTÍSTICO COMO ESTRATEGIA DE ENSEÑANZA-APRENDIZAJE EN EDUCACIÓN INFANTIL
}

Sandra Carbonell Mullor ${ }^{1}$

Resumen: El presente trabajo persigue comparar los patrones de conducta interpersonales que promueve el trabajo cooperativo frente al individual. Además, pretende comprobar si el aprendizaje cooperativo es una metodología válida en el aula de tres años de Educación Infantil. Para ello, se realizará una búsqueda bibliográfica y una propuesta práctica en la que se llevarán a cabo dos sesiones, dentro del área de Educación Plástica, una de trabajo cooperativo y otra de trabajo individual. Los datos obtenidos demostrarán que el aprendizaje cooperativo tiene unos mejores resultados.

Palabras clave: Aprendizaje Cooperativo, Aprendizaje individual, Educación Infantil, Interacción

Abstract: The aim of this undergraduate thesis is to compare the interpersonal behavioural patterns that are provoked by cooperative work versus individual work. Furthermore, it aims to verify whether cooperative learning is a valid methodology for classes of three year-old children. In order to do this, first, a review of the relevant literature will be carried out. Following this, a practical investigation will be conducted in which two sessions of Art class are analysed: one involving a cooperative activity and the other an individual activity. The data obtained shows that cooperative learning provide better results.

Keywords: Cooperative Learning, Individual Learning, Infant Education, Interaction.

\section{Introducción}

\subsection{Justificación}

Uno de los problemas que más preocupan a los profesionales del mundo educativo es el comportamiento individualista y competitivo de gran parte de la sociedad y, más que ello, que una de las principales causas de este tipo de conductas sea el propio sistema educativo.

Este individualismo que se crea desde los colegios, probablemente, es uno de los principales motivos de la deshumanización y la corrupción de hoy en día, de la incomunicación y el aislamiento que tenemos a pesar de estar en plena era de la comunicación con el mundo que nos rodea, incluso del progresivo deterioro de nuestras relaciones con la naturaleza.

\footnotetext{
${ }^{1}$ Universitat de València. sandracarbonell1995@gmail.com

Artículo recibido: 10 de septiembre de 2017; aceptado: 15 de octubre de 2017.
} 
Una de las soluciones primordiales para dicha problemática es cambiar el concepto que tenemos de Educación. Es por ello por lo que modificar los modelos de aprendizaje empleados debería ser una de nuestras prioridades, pues éstos no responden a la diversidad de alumnado que encontramos en el aula ni a las necesidades de los infantes.

En el presente trabajo investigaremos acerca del aprendizaje cooperativo, una metodología de aula que, como señala Candela, García y Traver [2001], favorece el aprendizaje de la solidaridad, sobre todo, desde sus componentes procedimentales y actitudinales. En concreto, nos situaremos en la etapa de Educación Infantil ya que, a pesar del egocentrismo que caracteriza a los niños y niñas a estas edades, es la base del desarrollo de la persona. El estudio se englobará en el área de aprendizaje artístico, puesto que está comprobado que las artes son una herramienta idónea para atender a la gran diversidad presente y dejar libertad de expresión al niño/a.

Vivimos en una sociedad cada vez más compleja, más dinámica y más interdependiente. En este contexto, tomar grandes decisiones en solitario es casi imposible. El verdadero reto de la Educación es desarrollar las habilidades sociales imprescindibles para hacer posible esta nueva era de colaboración y cooperación.

\subsection{Objetivos}

Con el presente trabajo se pretende reflexionar sobre cuáles son las diferencias y similitudes en los patrones de conducta interpersonales de los niños/as del aula de tres años a lo largo de una sesión de trabajo individual y otra de trabajo cooperativo en el área de plástica. Partiendo de esta premisa, los objetivos específicos planteados son los siguientes:

- Comprobar si el aprendizaje cooperativo es una metodología válida en Educación Infantil.

- Conoceryevaluar las diferencias entrela interacción con los compañeros/a[comunicación, empatía y conflicto] que promueve el aprendizaje individual y el que fomenta el aprendizaje cooperativo.

\section{Marco teórico}

A continuación, presentaremos de modo sintetizado la revisión de literatura realizada sobre el aprendizaje cooperativo, imprescindible para entender y justificar la práctica que más tarde se expondrá.

\subsection{Resultados obtenidos en diferentes estudios sobre el aprendizaje cooperativo}

Son numerosas las investigaciones realizadas a lo largo de la historia sobre el aprendizaje cooperativo. Entre los estudiosos de los elementos fundamentales del aprendizaje cooperativo podemos encontrar algunas tendencias o corrientes bien definidas y específicamente diferenciadas [Lobato 1997): 


\section{- El Learning Together de D.W. Johnson y R.T. Johnson.}

Según Johnson y Johnson [1999] la principal ventaja del aprendizaje cooperativo es la cantidad de procedimientos y actitudes positivas que promueve. Entre ellas destacan las siguientes:

Brindar al otro una ayuda más efectiva y eficaz.

a) Proporcionar al otro/a realimentación para que pueda mejorar el futuro desempeño de sus actividades y sus responsabilidades.

b] Desafiar las conclusiones del otro/a y razonar para favorecer una toma de decisiones de mayor calidad y una mayor comprensión de los problemas.

c] Influir en los esfuerzos del otro/a para alcanzar objetivos grupales.

d) Estar motivado/a para esforzarse por el beneficio mutuo.

e) Tener un nivel de excitación moderado, caracterizado por un bajo nivel de ansiedad y estrés.

\section{- El Student Team Learning de Slavin.}

Según Slavin [1992] tres son los elementos esenciales y característicos del método o de la técnica Student Team Learning de un aprendizaje cooperativo:

a) La recompensa del grupo.

b) La responsabilidad individual.

c] La misma oportunidad de éxito.

Finalmente, Slavin [1999] comenta que deberemos tener especialmente cuidado al trabajar de manera cooperativa, ya que existe un alto riesgo de que la implicación por parte de los miembros del equipo este muy desequilibrada. Es lo que denomina el efecto "polizón", en el cual unos componentes del grupo realizan gran parte del trabajo y otros/as poco o nada.

\section{- El Group Investigation de Sharan y Sharan}

En sus estudios destacan los aspectos de la interrelación grupal: la investigación, la interacción, la interpretación y la motivación intrínseca.

La interacción tiene por objeto la investigación, la repartición de los contenidos descubiertos y el placer de descubrirlos. Los problemas y los interrogantes son afrontados buscando junto a los otros/as los contenidos, interpretándolos, discutiéndolos y, por esto, estimulados por el interés de conocer. La motivación por conocer es suscitada por una curiosidad movida por interrogantes y problemas, y mantenida por la interacción y la confrontación. 


\section{- La Structural Approach de Kagan y Kagan}

En sus publicaciones (Kagan y Kagan, 1994) consideran como elementos claves del Structural Approach seis aspectos:

a] La estructura adaptada al interés u objetivos que se busquen.

b) Los principios fundamentales del aprendizaje cooperativo [interacción simultánea, igualdad de posibilidades, interdependencia positiva y la responsabilidad individual].

c] La construcción del grupo y del clima de la clase positivo para garantizar la calidad de las relaciones interpersonales.

d) El concepto de equipo como grupo con identidad con no más de cuatro miembros y en función de los intereses fijados.

e) La conducción de la clase siendo el docente planificador pero a la vez guía en el seguimiento de los procesos y los sujetos activos en el aprendizaje.

f] El aprendizaje y desarrollo de competencias sociales como eje para llevar a cabo el trabajo cooperativo.

\section{- La Complex Instruction de E. Cohen}

Elisabeth G. Cohen (1994) busca constatar que para llevar a cabo el aprendizaje cooperativo hace falta algo más que poner personas juntas para trabajar.

Tras la realización de diversas investigaciones descubre que hay un factor crucial en un grupo, es el estatus con que el sujeto entra al grupo. Es por ello, que en la construcción de los grupos es fundamental tener en cuenta esta característica. En cierta forma, todas las personas clasificamos a los demás, y a la vez nos sentimos clasificados por otros. No obstante, todos/as perciben que es mejor colocarse en un puesto alto que bajo.

Este estatus no se refiere únicamente a las cualidades intelectuales, tampoco es un problema de desigualdad social o de clasificación interna del grupo, sino que tiene un amplio espectro de variables personales, culturales y sociales. Es curioso, como en sus investigaciones constata que un estatus alto predice o coincide con un mayor aprendizaje adquirido al finalizar el trabajo grupal, por lo que resulta paradójico que buscando promover la igualdad el efecto sea el contrario y se marquen más las diferencias.

La autora recomienda que a la hora de planificar un grupo se tenga en cuenta este estatus. Bajo su punto de vista, las estrategias del aprendizaje cooperativo tienden a superar los límites de partida que amenazan los resultados que el método quiere conseguir. Para intentar que el efecto 
del estatus se reduzca al máximo es necesario establecer las siguientes condiciones: modificar los prejuicios del alumnado y el profesorado, preparar al alumnado a la cooperación por medio de la enseñanza de las competencias cooperativas específicas, organizar tareas complejas, dar a cada miembro el rol o tarea a desempeñar y finalmente evaluar y optimizar el trabajo en grupo.

\section{- La perspectiva del Collaborative Approach}

Tres de los aspectos fundamentales que la investigadora Cowie y sus colaboradores Smith, Boulton y Laver [1994] formulan atendiendo a los resultados obtenidos en la investigación sobre el trabajo en grupo como cambio social son los siguientes:

a) Los estudiantes deben tener experiencias de grupo no basadas solo en la amistad sino en grupos cooperativos.

b] Para formar grupos cooperativos es necesario enseñar a los estudiantes las habilidades de comunicación, de intercambio de informaciones, de trabajo eficaz compartiendo las cargas para alcanzar un objetivo común.

c] Una competencia absolutamente necesaria es la capacidad de saber afrontar y superar conflictos.

A continuación, comentaremos algunas de las conclusiones expuestas en diferentes trabajos de investigación llevados a cabo directamente en el aula de Educación Infantil.

En primer lugar, Zambrano [2014] tras realizar una propuesta con la intención de introducir al alumnado y habituarlo a trabajar de una manera cooperativa mediante la reorganización del aula y diferentes actividades a lo largo de tres semanas, extrae las siguientes conclusiones:

a) Para que trabajar mediante aprendizaje cooperativo resulte muy beneficioso, se debe solapar con una metodología más abierta y no por tanto ser un momento aislado dentro de una metodología tradicional. De ser así, puede provocar un conflicto interno en el niño/a.

b] Aprender cooperativamente va más allá de un simple trabajo en grupo, puesto que consigue que los infantes se sientan más incluidos en el grupo, se ayuden entre ellos/as y a la vez estén más motivados.

c) Aunque se trabaje por grupos el alumnado tiene la necesidad de competir, por lo que se aprecia la ayuda entre el grupo y la competitividad con el resto. Por ello es necesario variar mucho los componentes del grupo con el fin de quitar prejuicios.

d) Impartir este tipo de metodología desde un principio facilita que los niños/as adquieran el modo de trabajo al llegar a una edad. 
e) Los niños/as de Educación Infantil tienen una capacidad de retención mínima por lo que realizar una actividad con un objetivo concreto, donde deben intervenir todos/as es demasiado complejo.

Otro de los estudios realizados por Arbonies [2013], en el que se diseña una propuesta de aprendizaje cooperativo durante una semana en el aula de Educación Infantil, demuestra lo siguiente:

a] Los niños/as no han conseguido interiorizar del todo lo que es el trabajo en equipo cooperativo puesto que era difícil cambiar el concepto de trabajo individual. No obstante, se contemplaron bastantes avances con el poco tiempo que se dispuso.

b) Durante y después de las sesiones de aprendizaje cooperativo hablaban y dialogaban mucho más entre ellos/as. A pesar de que al principio tomar decisiones conjuntas y conversar era nuevo para los infantes se notó mucha más soltura e interacción con el paso de las sesiones. Entendieron muy bien el hecho de tener que estar de acuerdo para proseguir la actividad.

c] El respeto les resulto fácil ya que se trataba de una clase con muy buen ambiente.

d) Les atrae la idea de que cada uno tenga una responsabilidad.

e) Se cumplieron los objetivos de las actividades satisfactoriamente, encontrando mayor dificultad en aquellas en que la técnica cooperativa era más compleja.

En cuanto a las posibles dificultades que podemos encontrar en el método del aprendizaje cooperativo, Lobato [1998], muestra las siguientes:

a) Los ritmos y los niveles académicos diferentes.

b) El individualismo arraigado entre el alumnado.

c) La falta de preparación y apoyo del profesorado.

d) La dificultad en la evaluación.

e) La mentalidad de las familias pendientes únicamente de determinados aprendizajes.

Con todo ello podemos se puede concluir que, a pesar de que todavía queda mucho camino en la investigación sobre el aprendizaje cooperativo, y sobre todo en la aplicación de éste en Educación Infantil, la gran parte de los estudios realizados coincide en las grandes ventajas de aplicarlo en las aulas, a pesar de las dificultades que puede suponer. 


\section{Metodología}

A continuación, procederé a explicar cuál ha sido mi propuesta para conseguir los objetivos planteados al comienzo del trabajo.

\subsection{Centro escolar}

La intervención se ha llevado a cabo en un Colegio Público de Educación Infantil y Primaria de la ciudad de Valencia, con un entorno socio-económico medio-bajo. En concreto, en la clase de tres años de Educación Infantil, es decir, su primer curso en el colegio. El aula está compuesta por un total de once alumnos y alumnas, de los cuáles seis son niñas y cinco niños. En ella encontramos grandes diferencias entre el alumnado respecto al nivel tanto madurativo como académico. Se trata de un aula en la que tienen algunos rasgos de organización cooperativa, por ejemplo, la asignación de cargos semanales, pero que todavía no se han iniciado en la realización de actividades cooperativamente, por tanto, supone un gran reto para ellos/as.

\subsection{Participantes}

A pesar de que las sesiones planificadas para la intervención han sido realizadas por todos/ as los niños/as del aula, la observación se ha centrado únicamente en cuatro de ellos/as. Con el fin de que la muestra representará a la diversidad de la clase, se escogió cuatro sujetos con características y ritmos de aprendizaje muy diferentes. El alumnado escogido fue el siguiente:

Sujeto A: Una niña muy trabajadora, con un nivel madurativo muy avanzado para la edad que tiene. La mayoría de veces realiza las actividades propuestas en clase satisfactoriamente.

Sujeto B: Un niño con un retraso madurativo en comparación con el resto de niños/as del aula y con gran dificultad para concentrarse en la realización de las tareas que se le proponen. Tiene un retraso en el lenguaje y a menudo le cuesta obedecer. No obstante, es muy cariñoso y está totalmente incluido en el grupo.

Sujeto C: Una niña trabajadora, perfeccionista e independiente a la hora de trabajar. Tiene bastante carácter y le resulta muy fastidioso que otros niños/as le molesten o griten.

Sujeto D: Un niño con gran falta de habilidades sociales para comunicarse con los compañeros/ as, tendiendo siempre a utilizar la agresividad. En ocasiones presenta también un comportamiento bastante desafiante con el profesorado. Además, es muy nervioso y le cuesta mucho mantenerse concentrado en una actividad y hacerla con delicadeza. No obstante, a nivel de razonamiento es uno de los más avanzados de la clase e intelectualmente también.

\subsection{Fases}

Primeramente, se programó dos sesiones en el área artística: una de ellas de trabajo individual y otra de aprendizaje cooperativo.

La intervención se realizó en el periodo de prácticas, en concreto en el mes de mayo, durante dos 
sesiones de dos días diferentes pero consecutivos, para que al finalizarlas se pudiera reflexionar sobre ambas y tenerlas recientes. El tiempo que se dedicó a cada sesión fue de 45 minutos. No obstante, hay que contemplar que este tiempo no fue rígido sino flexible.

Respecto al planteamiento de las sesiones, y recordando que nos encontramos en un aula de tres años, se decidió que en ambas realizaran un dibujo libre, para que la comparación posterior fuera más objetiva y los factores externos que condicionaran la actividad fueran los mínimos posibles.

Primero se realizó el dibujo individual. La disposición fue en una mesa todos/as juntos/as, ya que esa es su manera habitual de trabajar. No se les indicó ninguna pauta específica.

En la siguiente sesión, se elaboró un dibujo cooperativamente con la técnica del "folio giratorio" [un alumno/a empieza la tarea y van pasando el folio al compañero/a de al lado siguiendo las agujas del reloj]. Como nunca habían utilizado esta forma de trabajo, y además no había grupos en clase establecidos, se siguió los siguientes pasos. Anteriormente a la actividad se elaboraron tres grupos, dos de cuatro componentes y uno de tres [los grupos eran heterogéneos]. A continuación, para iniciar la sesión se comunicó los grupos al alumnado y cada equipo se sitúo alrededor de una mesa, para facilitar un clima más apropiado. Una vez acomodados, cada grupo concordó cual iba a ser el tema de su dibujo. Se realizó el dibujo en un folio D3 hasta que estuvieron de acuerdo en que el dibujo estaba finalizado. En esta actividad se les orientó sobre el funcionamiento y se les insistió en la importancia de consultar a sus compañeros/as antes de tomar una decisión, ya que era la primera vez que la realizaban.

\subsection{Instrumentos para la recogida de datos}

Respecto a la evaluación y la recogida de información de ambas sesiones, se han utilizado tres herramientas. En primer lugar, mediante la observación durante el proceso de las actividades con una tabla de elaboración propia [Anexo 1]. El método de observación que se tomó como base es el inCLASS [The individualizad Classroom Assessment Scoring System] [Booren, Downer, Lima, Luckner y Pianta, 2010]. Se realizaron dos observaciones de 50 minutos. Cada observación se dividió en tres ciclos, ya que el rendimiento de los niños y niñas al principio de la sesión no es igual que al final, y en cada ciclo se observó alternativamente a los cuatro sujetos. A cada uno de ellos/ as se les dedicó un tiempo por ciclo de cuatro minutos, de los cuales dos y medio fueron para observar y el minuto y medio restante para completar la tabla. En la tabla se valora del uno al cuatro, siendo el cuatro el resultado más satisfactorio. No obstante, en el apartado de "conflicto con los compañeros/as" el cuatro indicará menor conflicto.

En segundo lugar, al acabar ambas sesiones se realizó una asamblea en la cual fueron planteadas algunas preguntas acerca de cómo se había sentido realizando ambas sesiones y cuál de ellas les había gustado más y por qué. Se utilizó una grabadora de voz para posteriormente transcribir las intervenciones y poder evaluarlas.

Finalmente, a pesar de que el objetivo principal de esta actividad no es el resultado, se analizó los 
dibujos realizados en ambas sesiones, ya que proporcionaban información acerca de la efectividad y la implicación del alumnado. Se elaboraron diferentes categorías con el fin de analizar más minuciosamente las características de éstos.

\section{Resultados}

En este apartado se expondrán los resultados obtenidos tras haber llevado a cabo las dos sesiones programadas.

En primer lugar se recogerán los datos obtenidos mediante la tabla de observación expuesta en el anterior apartado; a continuación, se analizarán los dibujos realizados por los sujetos tanto individual como cooperativamente; finalmente, se comentaran los resultados obtenidos en la asamblea final.

\subsection{Resultados de la observación}

A continuación, se expondrán los resultados de la observación realizada. En las gráficas se mostrará el porcentaje que ha obtenido cada sujeto en los diferentes números de la escala de valoración [1, 2, 3, 4]. Cada categoría tendrá dos tablas: una con los resultados del periodo de observación durante la realización del dibujo individual y otra del cooperativo.

\subsubsection{Comunicación con los compañeros/as}

En las gráficas 1 y 2 se muestran los porcentajes que han obtenido los diferentes sujetos en cada número de la escala de valoración empleada para la recogida de datos durante la observación en la categoría de "la comunicación entre compañeros/as". En ella se valoraba el grado en que el sujeto iniciaba y mantenía conversaciones con el resto de infantes durante la elaboración del dibujo. Como vemos en las figuras gráficas, mientras que en la sesión que trabajan individualmente el número predominante ha sido el 1, en la sesión cooperativa el mayor porcentaje se encuentra en el 3.

Fig. 1: Categoría 1. Sesión individual

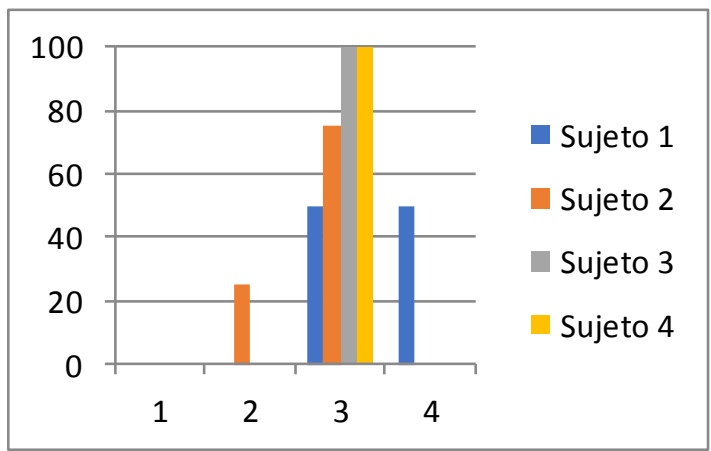

Fig. 2: Categoría 1. Sesión cooperativa

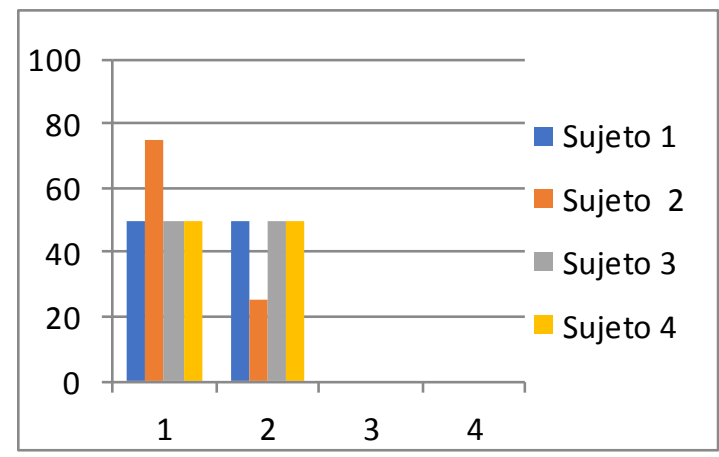




\subsubsection{Sociabilidad y empatía con los compañeros/as}

En las figuras 3 y 4 se muestran los porcentajes que han obtenido los diferentes sujetos en cada número de la escala de valoración empleada para la recogida de datos durante la observación en la categoría "sociabilidad y empatía con los compañeros/as". En ella se valoraba el grado en que el sujeto brindaba y recibía ayuda, motivaba al resto de infantes, respetaba el trabajo de sus compañeros/as y compartía afecto positivo. Como vemos en la gráfica, mientras que en la sesión que trabajan individualmente el número predominante ha sido el 1, en la sesión cooperativa el porcentaje se encuentra más repartido entre el 2,3 y el 4.

Fig. 3: Categoría 2. Sesión individual

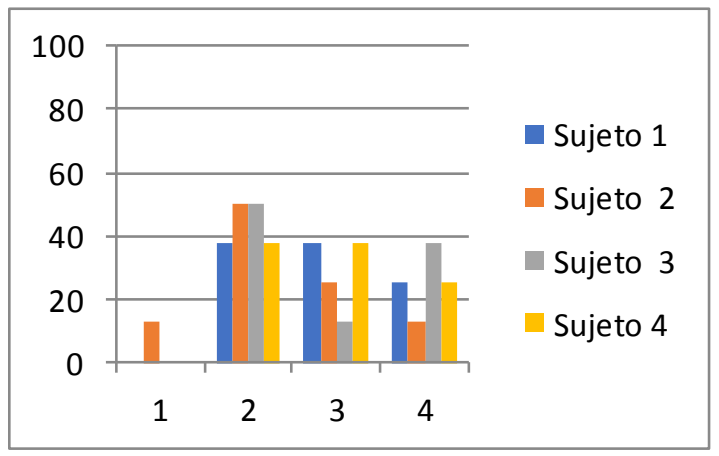

Fig. 4: Categoría 2. Sesión cooperativa

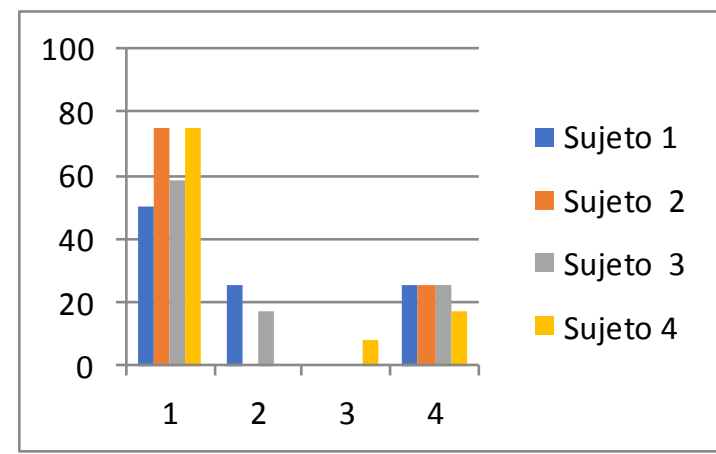

\subsubsection{Conflicto con los compañeros/as}

En las figuras 5 y 6 se muestran los porcentajes que han obtenido los diferentes sujetos en cada número de la escala de valoración empleada para la recogida de datos durante la observación en la categoría de "conflictos con los compañeros/as". En ella se valoraba el grado en que el sujeto mantenía confrontaciones con sus compañeros/as o presentaba comportamientos agresivos. Recordar que en este caso, la valoración era al revés, puesto que el 1 era el mejor resultado ya que representaba el menor grado de conflicto. Como se observa en las gráfica, el intervalo predominante en ambas sesiones es 1, no obstante, en el trabajo individual el sujeto 4 la mitad de sus valoraciones se encuentran en el grado 3.

Fig. 5: Categoría 3. Sesión individual

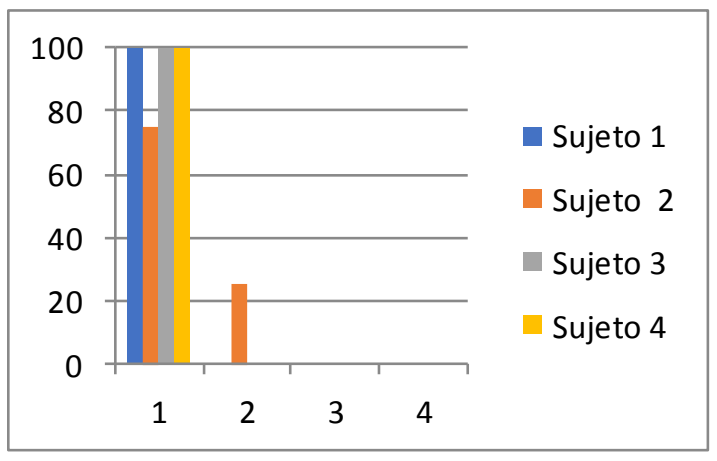

Fig. 6: Categoría 3. Sesión cooperativa

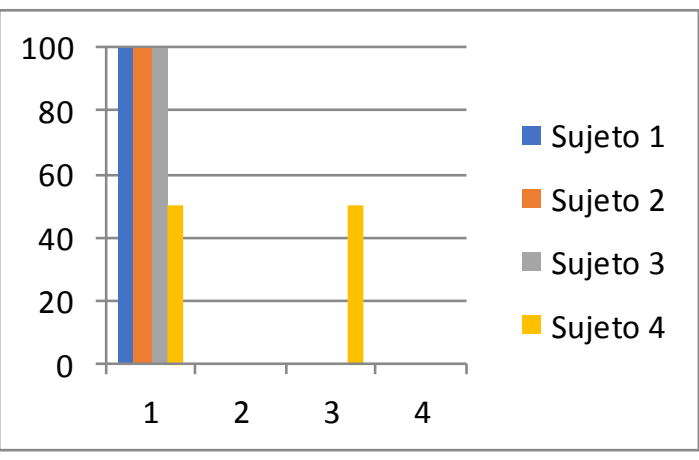




\subsection{Resultados de los dibujos}

Los dibujos realizados por los sujetos individualmente y cooperativamente con su respectivo grupo son los siguientes:

Dibujos individuales

Fig. 7. Dibujo sujeto 1

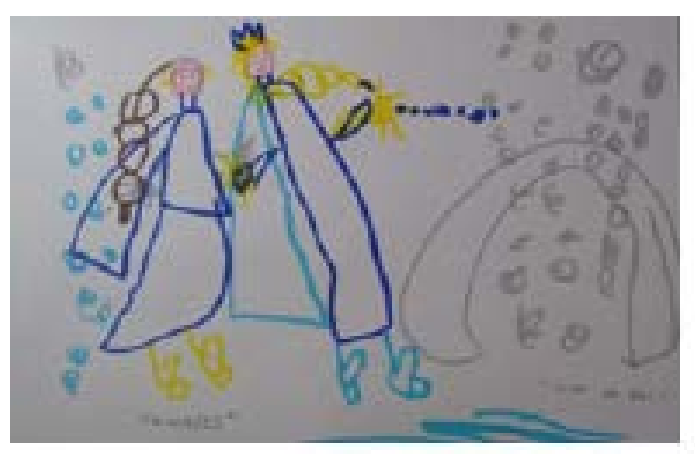

Fig. 9. Dibujo sujeto 3

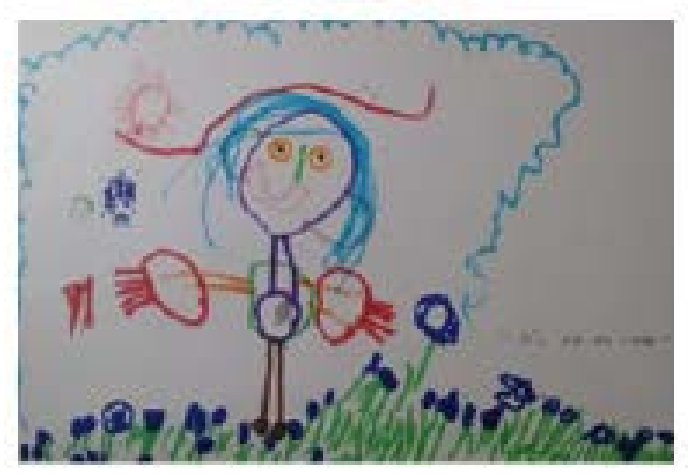

Fig. 8. Dibujo sujeto 2

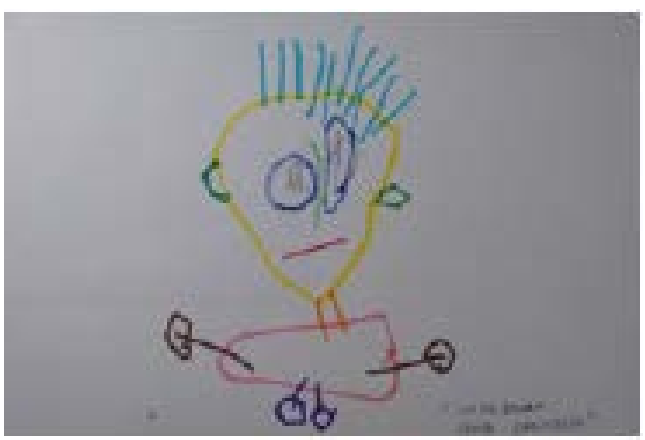

Fig. 10. Dibujo sujeto 4

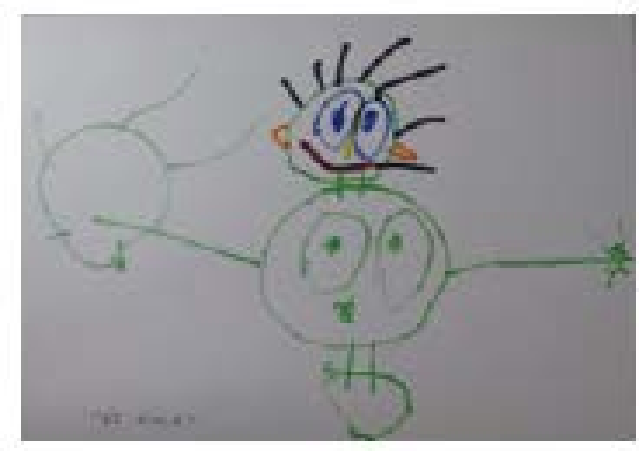

Dibujos cooperativos

Fig. 11. Dibujo equipo 1

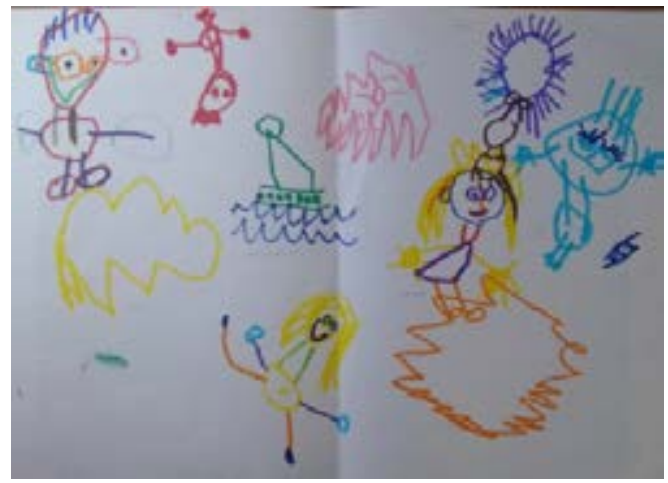

Fig. 12. Dibujo equipo 2

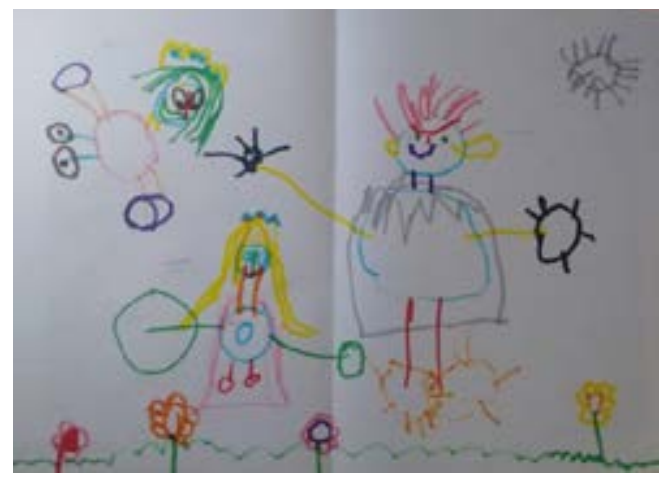


Fig. 13. Dibujo equipo 3

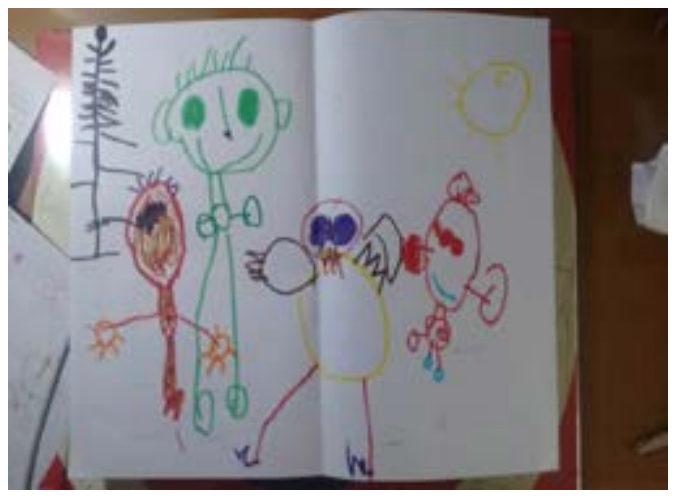

A continuación, en la tabla 1 se muestran las categorías construidas después de la realización de ambas sesiones:

Tabla 1: Categorías construidas.

\begin{tabular}{|l|l|}
\hline Categorías & Características \\
\hline Dimensión social del dibujo & Individual [IND], Cooperativo [COOP], Competitivo [COMP] \\
\hline Interacción individuo-entorno & $\begin{array}{l}\text { Hay elementos del entorno [SI] } \\
\text { No hay elementos del entorno [NO] }\end{array}$ \\
\hline Ubicación de la figura[s] humana & Centro [C], Ocupando diferentes espacios [OD] \\
\hline Afectividad & Hay afectividad [SI], No hay afectividad [No] \\
\hline
\end{tabular}

\subsubsection{Representación de los dibujos individuales}

En la tabla 2 se observa la descripción de los dibujos realizados individualmente por los sujetos de acuerdo con las categorías construidas.

Tabla 2: Análisis dibujos individuales.

\begin{tabular}{|l|l|l|l|l|}
\hline & $\begin{array}{l}\text { Dimensión social } \\
\text { del dibujo }\end{array}$ & $\begin{array}{l}\text { Interacción } \\
\text { individuo-entorno }\end{array}$ & $\begin{array}{l}\text { Ubicación de la figura } \\
\text { humana }\end{array}$ & Afectividad \\
\hline Dibujo 1 & COOP & SI & C & SI \\
\hline Dibujo 2 & IND & NO & C & NO \\
\hline Dibujo 3 & IND & SI & C & NO \\
\hline Dibujo 4 & IND & NO & C & NO \\
\hline
\end{tabular}




\subsubsection{Representación de los dibujos cooperativos}

En la tabla 3 se observa la descripción de los dibujos realizados cooperativamente por los sujetos de acuerdo con las categorías construidas.

Tabla 3: Análisis dibujos cooperativos.

\begin{tabular}{|l|l|l|l|l|}
\hline & $\begin{array}{l}\text { Dimensión social } \\
\text { del dibujo }\end{array}$ & $\begin{array}{l}\text { Interacción } \\
\text { individuo-entorno }\end{array}$ & $\begin{array}{l}\text { Ubicación de la } \\
\text { figura humana }\end{array}$ & Afectividad \\
\hline Dibujo 1 & COOP & Sl & OE & $\mathrm{SI}$ \\
\hline Dibujo 2 & COOP & Sl & OE & $\mathrm{SI}$ \\
\hline Dibujo 3 & COOP & Sl & OE & SI \\
\hline
\end{tabular}

\subsection{Resultados de la entrevista}

En la asamblea final realizada una vez acabada ambas sesiones, uno de los seis infantes que respondieron, es decir, un 16,66\%, escogió la realización del dibujo individual frente al cooperativo. El resto de alumnado, entre los cuales estaban los cuatro sujetos objetos de la presente investigación, un 83,3\%, explicó su preferencia del dibujo cooperativo frente al individual. La entrevista se encuentra transcrita en el Anexo 2. Los argumentos expuestos son los mostrados en la tabla 4:

Tabla 4: Argumentos a favor dibujo individual o cooperativo.

\begin{tabular}{|l|l|}
\hline Argumentos dibujo individual & $\begin{array}{l}\text { Argumentos dibujo cooperativo } \\
\text { "Porque me gusta estar al lado" } \\
\text { "Perquè sinó em ratllen el meu dibuix." }\end{array}$ \\
$\begin{array}{l}\text { "Perquè anem en una mesa, en una mesa otros i } \\
\text { en una mesa otros." } \\
\text { "Perquè així estoy con Laura y mis amigos." } \\
\text { "Perquè estoy a un costat i puedo parlar amb } \\
\text { ells." } \\
\text { "Con mis amigos, asi puedo dibujar a Spider-man } \\
\text { y a Hulk." }\end{array}$ \\
\hline
\end{tabular}

Mientras que el argumento principal del infante que defiende el dibujo individual viene relacionado con el conflicto que puede suponer el dibujo cooperativo entre los compañeros/as, aquellos/as que prefieren el dibujo cooperativo argumentan mediante la necesidad de afectividad, interacción y motivación. 


\section{Discusión}

A lo largo de este apartado procederemos a la discusión de los resultados. Para ello, se agruparán según las categorías utilizadas anteriormente, en relación con los objetivos del trabajo expuestos al inicio del documento.

\subsection{Comunicación con los compañeros/as}

De acuerdo con los resultados obtenidos durante la observación realizada, en la sesión cooperativa la comunicación entre el alumnado fue mucho mayor que en la individual (fig. 1 y 2). Además, este incremento se ha dado en todos los sujetos, a pesar de las características particulares de cada uno.

Se produjo una gran diferencia entre las conversaciones mantenidas durante el trabajo individual y el cooperativo. En la primera sesión [individual], las interacciones de los sujetos 2 y 4 , eran principalmente con la maestra, mientras que los sujetos 1 y 3 prácticamente no interactuaban puesto que son más maduras y realizaban el dibujo independientemente. Sin embargo, en la segunda sesión se vio mucha más interacción y comunicación entre ellos/as. El simple hecho de disponerlos en pequeños grupos y en círculo ya promovía una mayor interacción. Como señala Johnson y Johnson (1999) la disposición frente-frente es fundamental para promover una interacción positiva.

Los primeros minutos fueron claves en esta categoría, pues éstos son destinados principalmente a conversar para decidir qué dibujar. Las conversaciones se mantuvieron a lo largo de toda la sesión y la mayoría se encontraban relacionadas con el tema, lo que implica también mayor atención.

Además de todo ello, los dibujos realizados cooperativamente muestran claramente una mayor comunicación y sociabilidad, siendo únicamente un sujeto el que dibuja más de una persona en su dibujo individual (tabla 2 y 3 ) al contrario que en el cooperativo donde este aspecto está presente en todos. Comunicarse es una necesidad de los infantes, ya que como uno de los sujetos dice en la entrevista final, le gusta más el aprendizaje cooperativo porque así puede hablar con sus amigos/as.

En la misma línea se mueven los resultados de Arbonies [2013] quien explica que a lo largo de las sesiones cooperativas planteadas los infantes hablaban y dialogaban mucho más entre ellos/ as. Esto se trasladaba, conforme pasaba el tiempo, a otros ámbitos fuera de las actividades planificadas, por ejemplo, cuando debían escoger un rincón en el que jugar. El hecho de que estas habilidades de comunicación que se van desarrollando se trasladen a otros contextos resulta uno de los objetivos principales de este tipo de aprendizaje. Como señala Kagan y Kagan [1994], se considera un eje del aprendizaje cooperativo el desarrollo de competencias sociales. 


\subsection{Sociabilidad y empatía con los compañeros/as}

Según Johnson, Johnson y Holubec (1999) la interacción estimuladora es uno de los cinco elementos más importantes del aprendizaje cooperativo. Con ello, nos referimos al intercambio de opiniones de forma libre, la confianza con el resto del grupo, la motivación etc. Todo lo nombrado resulta indispensable para que el alumnado que compone el grupo se sienta valorado y respetado por el resto.

Analizando las gráficas de las figuras 3 y 4, las cuales recogen los resultados obtenidos en esta categoría durante la sesión individual y cooperativa, observamos que los infantes ofrecen más ayuda y motivan al resto de compañeros/as mientras realizan el dibujo cooperativo. Es verdad que los resultados tampoco han sido tan altos como esperábamos en dicha sesión, pero debemos tener en cuenta que nunca habían realizado nada similar y por tanto no tienen interiorizado lo que la interdependencia de finalidades positiva propia de la estructura de la actividad cooperativa. Señalar que, por su parte, el respeto del trabajo de los compañeros/as ha obtenido un porcentaje bastante alto en ambas sesiones ya que es un tema en que la tutora del aula insiste mucho día a día. Este aspecto lo destacan Kagan y Kagan [1994] quienes consideran que uno de los elementos fundamentales del aprendizaje cooperativo es la construcción del grupo y del clima de la clase para garantizar las relaciones interpersonales. En esta línea se mueven también los resultados de Arbonies [2013] quien afirma que el respeto les resultó fácil a los sujetos de su intervención puesto que se trataba de una clase con muy buen ambiente.

Además de todo lo comentado, si nos paramos a analizar los resultados de los dibujos realizados por los sujetos [tablas 2 y 3 ], mientras que en los dibujos individuales las figuras humanas representadas se encuentran justo en el centro del papel mostrando el egocentrismo propio de la edad, en el dibujo cooperativo las figuras humanas se encuentran más esparcidas por todo el espacio. Asimismo, al contrario que en los dibujos individuales donde solo dos sujetos dibujan elementos del entorno, en los dibujos cooperativos la interacción con los elementos que les rodean está representada en todas las producciones. También, la afectividad es mayor en los dibujos cooperativos pues son más las figuras dibujadas, hay mayor movimiento y expresión en sus caras.

Todo ello nos ayuda a reafirmar que el aprendizaje cooperativo promueve una mayor empatía y sociabilidad en los sujetos. Los infantes necesitan esta afectividad e interacción para desarrollarse, porque como muestran los argumentos expuestos por los sujetos en la entrevista final todos ellos/as buscan mayor contacto y cercanía con sus compañeros/as.

\subsection{Conflicto con los compañeros/as}

Los resultados de la observación de conflicto con los compañeros/as han sido bastante buenos en ambas sesiones, pues como hemos comentado anteriormente se trata de una clase pequeña y con un buen clima. No obstante, en ambas ha habido un pequeño porcentaje de conflicto. En la sesión individual, el sujeto 4 fue el que generó una pequeña discusión; como ya he dicho, se 
trata de un infante muy impulsivo y un poco agresivo, por lo que el conflicto, como suele suceder en el día a día del aula, se originó por estas características. No obstante, en la sesión del trabajo cooperativa nos sorprendió el buen comportamiento que mantuvo ya que no promovió ninguna discusión y mostró una actitud muy respetuosa.

En la sesión cooperativa las pequeñas confrontaciones vinieron sobretodo originadas por el hecho de tener que esperar y respetar el turno. Como explica Zambrano [2014] los niños/as tienen una capacidad de retención mínima, por lo que realizar una actividad donde tienen que intervenir todos/as y respetar los turnos es demasiado complejo al principio.

Para finalizar, resaltar un conflicto que surgió al principio de la sesión en el momento en que se tenían que poner de acuerdo sobre que dibujar. Dos miembros de un grupo querían hacer cosas diferentes y tuvieron una pequeña riña sobre cuál de la dos opciones harían. Sin embargo, fueron ellas mismas quienes finalmente resolvieron el conflicto y decidieron hacer las dos cosas escogiendo un tema en común. Como explican Boulton, Cowie, Laver y Smith, [1994] una competencia absolutamente necesaria es la capacidad de saber afrontar y superar conflictos.

\section{Conclusiones}

Como se ha demostrado en los resultados, el aprendizaje cooperativo promueve, frente al individual, una mayor interacción con los compañeros/as. Es verdad, que los resultados obtenidos no han sido quizás muy satisfactorios debido a diversos factores, entre ellos el gran arraigo de la cultura individualista y competitiva en el sistema educativo, pero el simple hecho de notar una mínima mejoría ya implica la idoneidad de un tipo de enseñanza-aprendizaje frente a otro.

Este balance positivo hacia el trabajo cooperativo no quiere decir que se deba eliminar por completo el proceso de enseñanza-aprendizaje individual sino que no hemos de centrar toda la atención en él. No podemos olvidar que para que el aprendizaje cooperativo funcione debe haber una gran responsabilidad individual por parte de los miembros del equipo.

La Educación, y en concreto la Educación Infantil, es la base de las personas y consecuentemente de la sociedad. En ella se busca un desarrollo integral de los seres humanos, por tanto, si tenemos una herramienta que puede ayudar a conseguir este objetivo por qué no utilizarla. Hemos comprobado como el aprendizaje cooperativo funciona notablemente en Educación Infantil, en concreto en tres años. A pesar de la escasez de tiempo empleado y de la corta edad, ha quedado demostrado como los resultados han sido óptimos.

Además, los resultados de la actividad, es decir, los dibujos realizados durante las sesiones han reflejado una mayor creatividad, empatía e interacción tanto entre personas como con el entorno. Cabe decir que los sujetos han elegido también este modo de trabajo frente al individual, por lo que el aumento de la motivación al enfrentarse a la tarea resulta evidente.

A menudo, tenemos miedo a lo nuevo, a romper con aquello establecido, a desconcertar la rutina de los niños y niñas del aula. No obstante, este conflicto que se crea en ellos/as es el precursor de un posterior aprendizaje. El aprendizaje cooperativo va más allá de un simple trabajo en grupo, 
o de un resultado final, busca desarrollar en el alumnado las habilidades inter e intra-sociales necesarias para vivir en sociedad, evitar ese individualismo que nos corrompe y esa competencia que nos ciega. Nos da la oportunidad de desarrollar lo mejor de nosotros/as mismos/as para dar entre todos/as la mejor solución. Permite sacar la singularidad de cada persona, otorgándole la posibilidad de que comparta aquello que mejor sabe hacer y mejore sus debilidades.

Al comenzar el recorrido del trabajo defendíamos el aprendizaje cooperativo, posiblemente sin argumentos, como una herramienta promotora de la interacción entre los niños/as frente a el individualismo, sin embargo, teníamos dudas sobre si funcionaría en Educación Infantil. Ahora, al acabar este trabajo, podemos afirmar que, este tipo de proceso enseñanza-aprendizaje es una de las soluciones a la estructura y los valores de la sociedad en la que vivimos, y por ello, cuanto antes lo empecemos a impartir en nuestras aulas mayores serán los resultados obtenidos. No es un proceso fácil, por supuesto, requiere de mucha implicación tanto por parte del profesorado como del alumnado, y mucha paciencia para llegar a los objetivos deseados, pero sin duda, ha quedado comprobado que merece la pena.

\section{Referencias bibliográficas}

Arbonies M. [2013). Propuesta para fomentar el aprendizaje cooperativo en Educación Infantil. Trabajo de fin de grado. Universidad Internacional de La Rioja, España. Recuperado de http:/ / www.svplaredo.es/Curso\%202015-2016/Metodologia/2013_05_27_TFM_ESTUDIO_DEL_ TRABAJO.pdf

Cowie, H., Smith, P. H., Boulton, M. y Laver, R. [1994]. Cooperation in the multiethnic classroom: The impact of Cooperative Group Work on social relationships in middle schools. London: David Fulton Publishers.

DECRET 38/2008, de 28 de març, del Consell, pel qual s'establix el currículum del segon cicle de l'Educació Infantil a la Comunitat Valenciana. [2008/3838]

Downer, J. T., Booren, L. M., Lima, O. K., Luckner, A. E., \& Pianta, R. C. [2010]. The Individualized Classroom Assessment Scoring System [inCLASS]: Preliminary reliability and validity of a system for observing preschoolers' competence in classroom interactions. Early childhood research quarterly, 25 (1), 1-16. Recuperado de http://www.sciencedirect.com/science/article/pii/ S0885200609000520

García, R., Traver, J. A., y Candela, I. [2001]. Aprendizaje cooperativo. Fundamentos, características y técnicas. Madrid: CCS.

Johnson, D.W. y Johnson, R. T. [1999). Aprender juntos y solos. Recuperado de http://www. terras.edu.ar/biblioteca/30/30JOHNSON-David-JOHNSON-Roger-Apendice.pdf 
Johnson, D. W., Johnson, R. T., \& Holubec, E. J. [1999). El aprendizaje cooperativo en el aula. Recuperado de http://s3.amazonaws.com/academia.edu.documents/33597188/EI_ aprendizaje_cooperativo_en_el_aula.pdf?AWSAccessKeyld=AKIAIWOWYYGZ2Y53UL3A\&Expir es=1497287396\&Signature=g9CIUMSljvwXDYD202w5cc\%2B4Z4\%3D\&response-contentdisposition=inline\%3B\%2Ofilename\%3DEl_aprendizaje_cooperativo_en_el_aula.pdf

Kagan, S. y Kagan, M. [1994]. The Structural Approach: Six keys to cooperative. En S. Sahran [Ed.]. Handbook of Cooperative Learning methods, pp. 115-133. Wesport, CT: Greenwood Press Lobato, C. [1997]. Hacia una comprensión del aprendizaje cooperativo. Revista de Psicodidáctica, 59 (4), 59-76. Recuperado de https://www.researchgate.net/publication/255663442_ Hacia_una_comprension_del_aprendizaje_coo-_perativo

Lobato, C. [1998]. El trabajo en grupo: aprendizaje cooperativo en secundaria. Bilbao: Servicio de Publicaciones de la Universidad del País Vasco

Slavin, R. E [1999]. Aprendizaje cooperativo: teoría, investigación y práctica. Recuperado de http:/ / apoclam.org/archivos-recursos-orientacion/Educacion\%2Oinclusiva/Estrategias/ slavin-el-aprendizaje-cooperativo.pdf

Sharan, Y. y Sharan, S. [1994]. Group Investigation in the cooperative classroom. En S. Sharan [Ed.], Handbook of Cooperative Learning methods [pp. 97-114]. Westpot, CT: Greenwood Press. Slavin, R. E. [1992]. When and why does Cooperative Learning increase achievement? Theoretical and empirical perspective. En R. Hertz-Lazarowitz y N. Miller [Eds.], Interaction in cooperative groups. The theoretical anatomy of group learning (pp. 145-173]. Nueva York: Cambridge University Press. Recuperado de http:/ / bit.ly/2iL7c2Y

Zambrano C. [2014]. El aprendizaje cooperativo en un aula multinivel de Educación Infantil. Trabajo de fin de grado. Universidad de Valladolid, España. Recuperado de https://uvadoc.uva. es/bitstream/10324/3489/1/TFG-B.310.pdf 


\section{Anexos}

\section{Anexo 1}

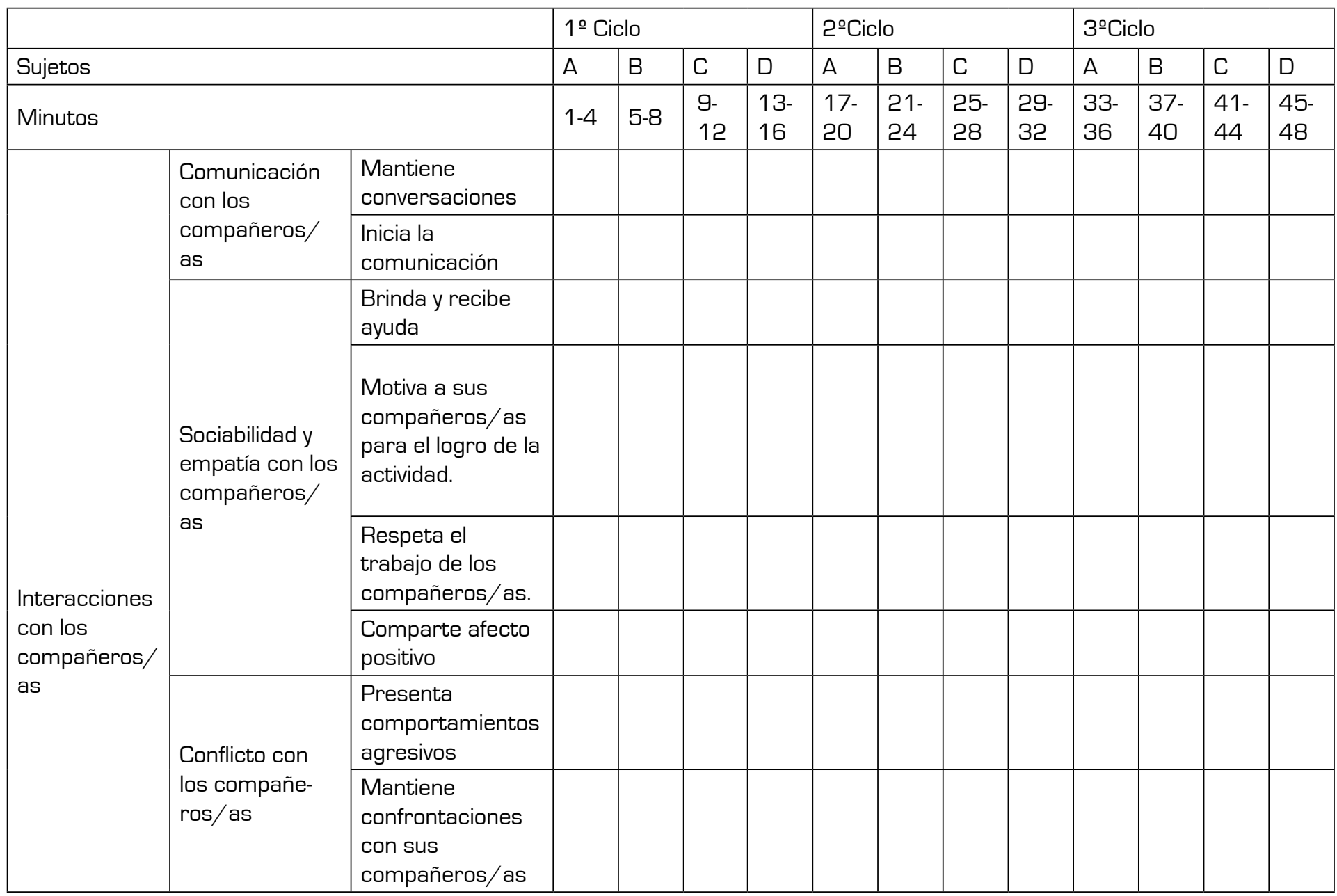




\section{Anexo 2}

\section{TRANSCRIPCIÓN ENTREVISTA}

S: Us acordeu del dibuix que vam fer ahir a soles i el que hem fet hui en grup?

A: Si!

S: I què us va agradar més dibuixar a soles o dibuixar en grups?

B: A mi dibuixar a soles, perquè sinó em ratllen el meu dibuix.

C: A mi dibuixar en grups.

S: Per què t'ha agradat més dibuixar en grups?

C: Perquè anem en una mesa, en una mesa otros i en una mesa otros.

S: Molt bé, i a tu S1?

A: En grup.

S: Per què t'ha agradat més dibuixar en grups?

A: Porque me gusta estar al lado

B: Si! Al lado de D!

S: I a tu S.3?

S4: Després jo vale?

S3: Perquè estoy a un costat i puedo parlar amb ells.

S4: I después yo vales?

S: I a tu sujeto 4? Que prefereixes en grup a soles?

S4: Estar en grupo.

S: En grup per què sujeto 4 ?

S4: Perquè així estoy con sujeto 2 y mis amigos.

S: Molt bé S4. I a tu S2? Què t’ha agradat més fer el dibuix en grup o a soles?

O: Yo a spider-man.

S: A val, i què t'ha agradat més fer el dibuix en grup o a soles?

O: A mi dibuixar a Spider-man.

S: Molt bé. Però prefereixes dibuixar-lo amb els teus amics/es o tu a soles?

O: Con mis amigos, asi puedo dibujar a Spider-man y a Hulk. 